HortSCIENCE 27(5):430-432. 1992.

\title{
Hydrotropism in Pea Roots in a Porous-tube Water Delivery System
}

\author{
Hideyuki Takahashi \\ Institute of Genetic Ecology, Tohoku University, 2-1-1 Katahira, Sendai \\ 980, Japan
}

\author{
Christopher S. Brown and Thomas W. Dreschel \\ Bionetics Corporation, Mail Code Bio-3, Kennedy Space Center, \\ FL 32899
}

\section{Tom K. Scott \\ Department of Biology, University of North Carolina at Chapel Hill, Chapel Hill, NC 27599}

Additional index words. Pisum sativum, 'Alaska' pea, agravitropic mutant, gravitropism, microgravity, nutrient delivery

\begin{abstract}
Orientation of root growth on earth and under microgravity conditions can possibly be controlled by hydrotropism-growth toward a moisture source in the absence of or reduced gravitropism. A porous-tube water delivery system being used for plant growth studies is appropriate for testing this hypothesis since roots can be grown aeroponically in this system. When the roots of the agravitropic mutant pea ageotropum (Pisum sativum L.) were placed vertically in air of $91 \%$ relative humidity and 2 to 3 $\mathrm{mm}$ from the water-saturated porous tube placed horizontally, the roots responded hydrotropically and grew in a continuous arch along the circular surface of the tube. By contrast, normal gravitropic roots of 'Alaska' pea initially showed a slight transient curvature toward the tube and then resumed vertical downward growth due to gravitropism. Thus, in microgravity, normal gravitropic roots could respond to a moisture gradient as strongly as the agravitropic roots used in this study. Hydrotropism should be considered a significant factor responsible for orientation of root growth in microgravity.
\end{abstract}

The use of a porous tube is under consideration as a part of water-nutrient delivery systems for the growth of plants in microgravity conditions (Dreschel and Sager, 1989;

Received for publication 5 Aug. 1991. Accepted for publication 4 Feb. 1992. Supported by a fund from the Yamada Science Foundation, Osaka, Japan, and a grant from the Institute of Space and Astronautical Science, Sagamihara, Japan, to H.T. and by a NASA grant (NAGW-1842) and a subcontract (no. 837-907-7) from Bionetics Corp. to T.K.S. Mention of brand name does not imply endorsement of the products by NASA or The Bionetics Corp. The cost of publishing this paper was defrayed in part by the payment of page charges. Under postal regulations, this paper therefore must be hereby marked advertisement solely to indicate this fact.
Koontz et al., 1990; Morrow et al., 1989; Wright et al., 1988). A major concern regarding the microgravity environment is the orientation of root growth in the absence of gravitropic responses due to weightlessness. Although not much attention has been paid to the hydrotropic responses of roots for many decades, roots have been reported to grow toward a moisture source (Darwin, 1880; Hooker, 1915; Knight, 1811; Sachs, 1872). A recent study using the agravitropic pea mutant ageotropum reported that roots show a positive hydrotropism in the absence of gravitropism (Takahashi and Suge, 1991). When the roots are capable of responding gravitropically, as in the case of nonmutant plants, hydrotropic and gravitropic responses interact with one another, resulting in a greater 


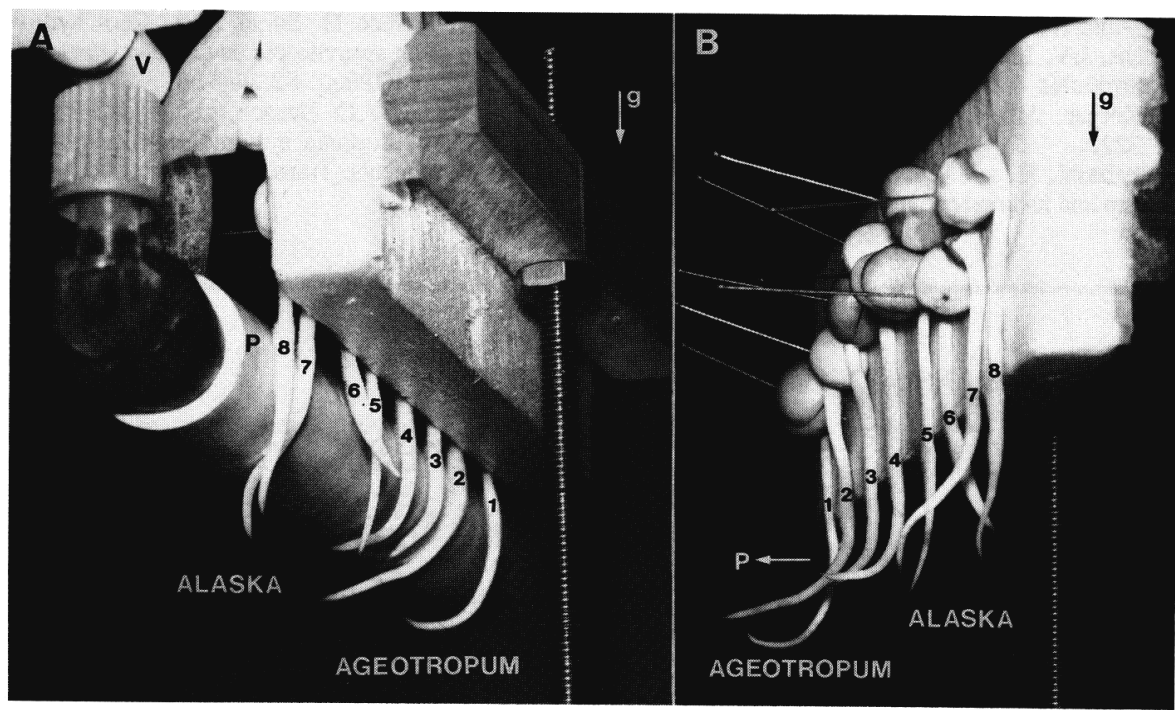

Fig. 1. Typical hydrotropic responses of 'Alaska' and ageotropum pea roots. Photographs were taken from the right with the porous tube (A) and from the left without the porous tube (B) $24 \mathrm{~h}$ after the start of the experiment. Roots 1-4 are ageotropum and 5-8 are 'Alaska' peas. (P) porous tube; (V) valve connected to a hypodermic syringe to pull water and to remove air; vertical arrow and (g) direction of gravitational vector. The porous tube was removed in B for the photograph; the side where the tube was is indicated by $\mathrm{P}$ and a horizontal arrow.

Table 1. Relative humidities ( $\mathrm{RH})$ at various distances from the porous tube. The RH was measured for both horizontal (sideways from the tube) and vertical planes (below the tube) 3,6 , and $12 \mathrm{~h}$ after the start of the experiment. Data represent the average of three time points $\pm \mathrm{SD}(\mathrm{n}=3)$.

\begin{tabular}{ccc}
\hline \hline $\begin{array}{c}\text { Distance from } \\
\text { porous tube } \\
(\mathrm{mm})\end{array}$ & $\begin{array}{c}\text { RH in } \\
\text { horizontal } \\
\text { plane } \\
(\%)\end{array}$ & $\begin{array}{c}\text { RH in } \\
\text { vertical } \\
\text { plane } \\
(\%)\end{array}$ \\
\hline 3 & $90.9 \pm 0.7$ & $91.5 \pm 0.7$ \\
10 & $84.6 \pm 1.0$ & $89.0 \pm 0.6$ \\
30 & $83.2 \pm 0.9$ & $87.9 \pm 0.6$ \\
50 & $82.6 \pm 0.8$ & $85.3 \pm 1.8$ \\
\hline
\end{tabular}

hydrotropic response as the degree of gravistimulation is lessened (Takahashi and Scott, 1991; Takahashi and Suge, 1991). These observations indicate that the orientation of root growth under microgravity conditions in space may well be controlled by a hydrotropic response. The porous-tube system is appropriate for testing hydrotropism. Since roots can be grown under atmospheric conditions in the system, the moisture gradient created by the tube is one factor responsible for the regulation of their growth orientation.

In this study, we have used a porous tube as a hydrostimulant and compared the hydrotropic response in roots of the agravitropic mutant pea ageotropum (Blixt et al., 1958) to that of the normal gravitropic 'Alaska' pea. A hydrophilic ceramic porous tube with pores $2 \mu \mathrm{m}$ in diameter $(12.5 \mathrm{~cm}$ long $\times 16 \mathrm{~mm}$ in diameter; Osmonics, Minnetonka, Minn.) was filled with distilled water. A $10-\mathrm{ml}$ syringe attached to one end of the tube was used to siphon water from a reservoir at the other end. When all the air was removed from the system by this means, the tube remained filled with water by the capillary action. To maintain a full system, evaporation of water through the pores was replenished at the reservoir; that is, water moved from the holding container and was maintained as an unbroken column as water evaporated from the tube. The saturated tube was placed horizontally in the middle of a 5.9-liter acrylic chamber $(16.5 \times 20.3 \times$ $17.5 \mathrm{~cm}$ ) with an inlet and an outlet for air flow. Moisture tensions of water at the top and at the bottom of the tube were -289.4 and $-96.5 \mathrm{~Pa}$, respectively. These water tensions did not vary throughout the experiments, as the tube was full of water at all times. To maintain $\approx 91 \%$ relative humidity (RH) 2 to $3 \mathrm{~mm}$ from the tube, where root tips were located, room air was filtered through $\mathrm{CaCl}_{2}$ and introduced into the chamber. For the inlet of air, two $15-\mathrm{cm}$ plastic pipettes with 10 holes each, serving as manifolds, were placed above and below the tube; an aquarium pump and split tubing connected to the pipettes was used to move air at rates between 50 to $300 \mathrm{ml} \cdot \mathrm{min}^{-1}$. Two outlet pipettes, like those noted above, were placed on the opposite sides of the inlet pipettes. Humidity in the chamber was controlled by changing the amount of $\mathrm{CaCl}_{2}$ used and/or the rate of air flow because the $\mathrm{RH}$ of room air was at $61.4 \% \pm 4.6 \%$ (mean $\pm \mathrm{SD}$ ) among experiments. RH and temperature at different places in the chamber was monitored by an HMI 31 humidity and temperature indicator with a HMP 36/0904 probe (Vaisala Sensor Systems, Helsinki, Finland). The sensor had been calibrated by the manufacturer 1 month before use; recalibration showed that no change had occurred. Experiments were performed at $22 \pm 1 \mathrm{C}$ and under continuous white light at the fluence rate of $15 \mu \mathrm{mol} \cdot \mathrm{m}^{-2} \cdot \mathrm{s}^{-1}$, obtained from four white fluorescent tubes (F40CW/RS/EWII, Philips, Bloomfield, N.J.) placed $2 \mathrm{~m}$ above the roots. No thermal gradients were detected at the distance between 2 to $3 \mathrm{~mm}$ and $50 \mathrm{~mm}$ from the porous tube in the chamber, although the surface temperature of the tube was not measured.

Seeds of 'Alaska' and ageotropum peas were germinated on wet filter paper in a closed container at $23 \pm 1 \mathrm{C}$ in darkness. When the seedlings were 2 to 2.5 days old, straight roots of both pea varieties, 25 to $30 \mathrm{~mm}$ in length, were suspended vertically by securing the cotyledons to a Styrofoam plate wrapped with moist paper (Whatman 40; Whatman International, Maidstone, England) using a 38-mm-long insect pin. The root-holding assembly was then placed by the side of the porous tube so that the root tips were located 2 to $3 \mathrm{~mm}$ from the tube, where the RH was preset at $\approx 91 \%$.

The $\mathrm{RH}$ gradient established in the chamber was not linear over a distance of $50 \mathrm{~mm}$ in either the horizontal or vertical plane (Table 1). By far, the largest gradient occurred within $10 \mathrm{~mm}$ of the porous tube in the horizontal plane. The RH at 2 to $3 \mathrm{~mm}$ from the tube,. where the roots were placed, remained at $\approx 91 \%$, and the gradient beyond that point decreased but was stable during the entire period of the experiment. In this moisture gradient environment, roots of ageotropum pea showed unequivocal positive hydrotropism 22 to $24 \mathrm{~h}$ after the start of stimulation by curving toward the porous tube, whereas 'Alaska' pea roots curved slightly toward the tube (curvature $91.5 \pm$ $5.1^{\circ}$ and $10.0 \pm 2.2^{\circ}$, respectively; difference significant at $P<0.01$, two-tailed Student $t$ test, $\mathrm{n}=18$ ) but often resumed vertical growth. Roots from both types of peas first grew downward with an accompanying nutation-like movement and then began to curve sideways, toward the porous tube (Fig. 1), between 3 and $4 \mathrm{~h}$ from the start of the stimulation, as analyzed from time-lapse video images (data not shown). Roots of ageotropum pea (roots 1-4) grew continuously along the round surface of the porous tube. By 24 $\mathrm{h}$ after the start of hydrostimulation, the root tips reached the opposite side of the tube and even curved upward along the opposite side of the tube as demonstrated by roots 1 and 4 (Fig. 1). Although the basal region of the root touched the surface of the porous tube, mechanical contact was not involved with the initiation or continuation of the hydrotropic response. Ageotropum roots curved continuously without mechanical contact between the responding region of the root and the porous tube (Fig. 1A). In 'Alaska' pea (roots 5-8), two roots showed only a slight and transient curvature toward the porous tube (roots 7 and 8), while two roots grew only vertically (root 5) or even curved away from the tube (root 6). The normal weak hydrotropic response of 'Alaska' pea is most likely due to an interacting or a counteracting effect of the gravitropic mechanism in the root. Initially, vertical roots curved sideways toward the hydrotropic. stimulant. However, gravitropism overcame this initial response as the roots grew more horizontally and the intensity of gravistimulation was accordingly increased to a sufficient threshold to cause 
growth to return to a vertical orientation (Takahashi and Scott, 1991; Takahashi and Suge, 1991).

Because ageotropum pea differs genetically from 'Alaska' pea, we were concerned whether 'Alaska' pea roots are ahydrotropic or not. However, we found that 'Alaska' pea roots responded hydrotropically similarly to ageotropum roots when the gravitropic response was nullified by rotating on a horizontal clinostat (data not shown). The observation suggests that the lesser hydrotropic response of 'Alaska' pea roots resulted from a counteracting effect by gravitropism. Permanent growth of roots downward or away from the moisture source often took place and was most likely due to a loss of turgor on the dry side of the root (Hooker, 1915; Takahashi and Suge, 1991). Such roots usually recover a positive hydrotropic response (Takahashi and Suge, 1991), although the occasional root remained straight or curved away from the moistened source as a result of the increased distance of hydrostimulant as well as the physiological status of the root (Fig. 1).

The results of this study indicate that the hydrotropic response may be a significant determining factor responsible for root orientation in a porous-tube water delivery system under microgravity. A reasonable hypothesis is that, in microgravity, normal gravitropic roots very likely behave hydrotropically, as did the agravitropic roots shown in this study. Because root hydrotropism occurs in darkness (Jaffe et al., 1985; Takahashi and Scott, 1991), roots may also respond to hydrostimulation for the control of root orientation in a dark outer space growing unit where light will have to be excluded for various reasons. Further, the root hydrotropism phenomenon in microgravity may be simulated under prevailing gravity on earth by the use of the agravitropic pea. mutant ageotropum.

\section{Literature Cited}

Blixt, S., L. Ehrenberg, and O. Gelin. 1958. Quantitative studies of induced mutations in peas. I. Methodological investigation. Agr. Hort. Gen. $16: 238-250$.

Darwin, C. 1880 . The power of movement in plants. John Murray, London.

Dreschel, T.W. and J.C. Sager. 1989. Control of water and nutrients using a Porous tube: A method for growing plants in space. HortScience 24:944-947.

Hooker, H.D., Jr. 1915. Hydrotropism in roots of Lupinus albus. Ann. Bot. 29:265-283.

Jaffe, M.J., H. Takahashi, and R.L. Biro. 1985. A pea mutant for the study of hydrotropism in roots. Science 230:445-447.

Knight, T. 1811. On the causes which influence the direction of the growth of roots. Philosophical. Trans. Royal Soc. London. Ser. B. Biol. Sci. 2:209-219.

Koontz, H.V., R.P. Prince, and W.L. Berry. 1990. A porous stainless steel membrane system for extraterrestrial crop production. HortScience 25:707.

Morrow, R.C., R.J. Bula, R.B. Corey, T.W. Tibbitts, and E.E. Richards. 1989. A porous-tube nutrient delivery system for plant growth in space.
Amer. Soc. Gravitationai Space Biol. Bul. 2:35. (Abstr.)

Sachs, J.V. 1872. Ablenkung der Wurzel von ihrer normalen Wachstumsrichtung durch feuchte Köper. Arb. D. Bot. Inst. Würzburg 1:209222.

Takahashi, H. and T.K. Scott. 1991. Hydrotropism and its interaction with gravitropism in maize roots. Plant Physiol. 96:558-564.

Takahashi, H. and H. Suge. 1991. Root hydrotropism of an agravitropic pea mutant, ageotropum. Physiol. Plant. 82:24-31.

Wright. B.D.. WC. Bausch. and W.M. Knott. 1988. A hydroponic system for microgravity plant experiments. Trans. Amer. Soc. Agr. Eng. 31:440-446. 